\title{
A novel oral camptothecin analog, gimatecan, exhibits superior antitumor efficacy than irinotecan toward esophageal squamous cell carcinoma in vitro and in vivo
}

Jianling Zou', Shuang Li', Zuhua Chen', Zhihao Lu', Jing Gao', Jianyin Zou², Xiaoting Lin', Yanyan Li', Cheng Zhang ${ }^{1}$ and Lin Shen ${ }^{1}$

\begin{abstract}
Esophageal squamous cell carcinoma (ESCC) is a frequently diagnosed and deadly malignancy with few standard therapeutic options. Camptothecins are considered one of the most promising antitumor drugs. A modified lipophilic analog, gimatecan, was synthesized as a novel oral camptothecin and showed impressive effects in various tumors, but its therapeutic efficacy and mechanisms in ESCC remain unclear. This study investigated the antitumor efficacy and mechanisms of gimatecan in ECSS both in vitro and in vivo. Using ESCC cell lines, cell line-derived xenografts and patient-derived xenografts models, we evaluated gimatecan's inhibition of tumor growth, and compared its antitumor efficacy with that of irinotecan. Topoisomerase I function and expression were assessed using the DNA relaxation assay and Western blotting, respectively. DNA damage was evaluated by Western blotting. Cell cycle progression and cell apoptosis were assessed using flow cytometry and Western blotting. Gimatecan could significantly suppress tumor growth in vivo and inhibit tumor cell proliferation in vitro, which was superior to irinotecan. Gimatecan suppressed the function and expression of topoisomerase I. It also caused DNA damage and activated the phosphorylation of multiple checkpoint gatekeepers, such as ATM, ATR, BRCA1, H2AX, CHK1, CHK2, and p53. It induced S phase arrest, enhanced the expression of p21 WAF1/CIP , and suppressed the expression of CDK2 and cyclin A. Induction of apoptosis was accompanied by increases in Bax, cleaved-caspase 3 activation, cleaved-caspase 9 induction, and a decrease in Bcl-2. The molecular and phenotypic changes induced by gimatecan were stronger than that of irinotecan. In ESCC, gimatecan suppressed the expression and function of topoisomerase I, induced DNA damage and intra-S phase cell cycle arrest, and resulted in apoptosis. And the results suggest that gimatecan has higher potency in inhibiting ESCC tumor growth than irinotecan, providing a rational novel therapeutic strategy for future clinical evaluation.
\end{abstract}

Correspondence: Cheng Zhang (qenya@163.com) or

Lin Shen (linshenpku@163.com)

${ }^{1}$ Department of Gastrointestinal Oncology, Key laboratory of Carcinogenesis and Translational Research (Ministry of Education), Peking University Cancer Hospital \& Institute, Beijing, China

2Department of Otolaryngology, Otolaryngology Institute of Shanghai Jiao Tong University, Shanghai Jiao Tong University Affiliated Sixth People's Hospital, Shanghai, China

The authors contributed equally: Jianling Zou and Shuang Li.

Edited by A. Stephanou

\section{Introduction}

Esophageal cancer (EC) is the fourth most commonly diagnosed and the most fatal cancer in China ${ }^{1}$. Esophageal squamous cell carcinoma (ESCC) is the predominant histological type of EC, comprising more than $95 \%$ of all EC cases ${ }^{2}$. ESCC is considered an aggressive malignancy due to the poor prognosis and high mortality rate. Most

\section{(c) The Author(s) 2018}

(c) (i) Open Access This article is licensed under a Creative Commons Attribution 4.0 International License, which permits use, sharing, adaptation, distribution and reproduction c. in any medium or format, as long as you give appropriate credit to the original author(s) and the source, provide a link to the Creative Commons license, and indicate if changes were made. The images or other third party material in this article are included in the article's Creative Commons license, unless indicated otherwise in a credit line to the material. If material is not included in the article's Creative Commons license and your intended use is not permitted by statutory regulation or exceeds the permitted use, you will need to obtain permission directly from the copyright holder. To view a copy of this license, visit http://creativecommons.org/licenses/by/4.0/. 
patients that are diagnosed with locally advanced or metastatic ESCC at the time of initial diagnosis ${ }^{3,4}$ are unable to undergo radical surgery, so the mainstays of treatment for these patients are radiation therapy and chemotherapy. However, the prognosis for patients with ESCC is still poor, with a 5-year survival rate of only about $20 \%{ }^{3}$. Therefore, it is crucial to identify the novel therapeutic alternatives or agents for patients with ESCC.

Due to their ability to disturb the catalytic cycle of DNA topoisomerase I, camptothecins are among the most promising antitumor drugs. They stabilize the covalent enzyme-DNA complex (cleavable complex) by forming a drug-enzyme-DNA complex during DNA synthesis, which is non-lethal and reversible. However, this causes the formation of irreversible double-stranded DNA breaks when a DNA replication fork collides with the cleavable complex ${ }^{5,6}$, Camptothecins can show preferential or selective toxicity to proliferating cells, particularly tumor cells, which are highly proliferative. As a result, the camptothecin analogs topotecan and irinotecan have already been widely used to treat several solid tumors, including colorectal carcinoma ${ }^{7,8}$ and lung cancer ${ }^{9-12}$. Recently, irinotecan has also shown promising results for the treatment of advanced ESCC ${ }^{13-15}$.

Although existing camptothecins have shown good tolerance and activity, a low therapeutic index is still the main disadvantage of clinical applications, which is largely attributed to the lability of the drug-enzyme-DNA complex and instability of the lactone ring. Consequently, research has aimed to enhance the antitumor efficacy, including modification of the molecular structure to induce topoisomerase I-mediated DNA cleavage and/or to stabilize the drug-enzyme-DNA complex ${ }^{16,17}$. The novel modified lipophilic analog gimatecan has been developed on the basis of this rationale, with the substitution at position C-7 by lipophilic chains ${ }^{18,19}$. This modification enhances rapid agent intake and stable drug interactions with intracellular targets ${ }^{20,21}$, and also allows the oral administration of gimatecan, which has shown advantages over oral topotecan in terms of the antitumor efficacy and therapeutic index in preclinical studies of non-small lung cancer and colon carcinoma ${ }^{22,23}$.

However, there has been no research into the application of gimatecan for ESCC, and the mechanism through which gimatecan suppresses proliferation of tumors remains unclear. This research assessed the antitumor efficacy of gimatecan and investigated its mechanism in ESCC.

\section{Results}

Gimatecan inhibits tumor proliferation of ESCC in vivo

To evaluate the antitumor activity of gimatecan in vivo, five cases of PDX models were selected and treated with saline containing 10\% DMSO (control) or gimatecan for
3 weeks. Compared to the control group, tumor growth was significantly suppressed in gimatecan-treated groups (TGIs were $94 \%, 136 \%, 112 \%, 105 \%$, and $81 \%$ in five cases, all $p<0.01$ ) (Fig. 1a-e). A representative image of PDX1's tumor at the end of treatment is shown in Fig. 1f.

\section{Gimatecan shows antitumor effects that are superior to irinotecan}

The capacity of gimatecan to inhibit cell proliferation was assessed in ESCC cell lines EC-109, KYSE450, KYSE140, KYSE-510, TE-1, and TE-10 with gradient dilutions for $48 \mathrm{~h}$, compared with irinotecan. Gimatecan showed strong inhibition in a dose-dependent manner at nanomolar concentration (Fig. 2a), which was lower than that of irinotecan (micromole level, Fig. 2b). Similarly, the IC50 of gimatecan in all chosen cell lines ranged from 4.9 $\pm 0.47 \mathrm{nM}$ to $39.6 \pm 0.32 \mathrm{nM}$, which were lower than that of irinotecan $(8140 \pm 366-37,680 \pm 521 \mathrm{nM})$, demonstrating that gimatecan inhibited cell proliferation of ESCC more effectively than irinotecan (Fig. 2c).

We then compared the antitumor effects of gimatecan to those of irinotecan in vivo. ESCC xenografts derived from cell lines Eca-109/KYSE-450 as well as two PDX models were used to evaluate the antitumor effects of gimatecan and irinotecan in vivo (Fig. $2 \mathrm{~d}-\mathrm{g}$ ). The results showed that gimatecan had a superior antitumor effect than irinotecan, particularly in the KYSE-450 xenograft (TGI $=90 \%$ vs. $52 \%, p<0.05)$ and PDX6 (TGI $=101 \%$ vs. $74 \%, p<0.05)$. The Eca-109 xenograft (TGI $=89 \%$ vs. $66 \%, p=0.06$ ) and PDX7 (TGI $=94 \%$ vs. $73 \%, p=0.08$ ) also showed greater inhibition of the tumor. Moreover, the given dosage did not cause significant loss of body weight.

\section{Gimatecan reduces topoisomerase I specific activity}

To explore the mechanisms of gimatecan, we first studied its inhibition of topoisomerase I using the DNA relaxation assay, compared with irinotecan. Topoisomerase I specific activity was inhibited by gimatecan in Eca109 and KYSE-450 cells at $10 \mathrm{nM}, 20 \mathrm{nM}$, and $40 \mathrm{nM}$ (Fig. 3a, b). Untreated cells had dominant proportions of relaxed DNA, while gimatecan-treated cells displayed a high ratio of the supercoiled form, indicating the loss of topoisomerase I activity. However, irinotecan treated cells at the same concentration mainly displayed relaxed DNA form. To verify the mechanisms, we increased the dosage of irinotecan, and found that irinotecan could also inhibit topoisomerase I specific activity in a dose- and timedependent manner in both cell lines (Supplement Fig. 1a, b). Such as, when treated with $40 \mu \mathrm{M}$ irinotecan for $2 \mathrm{~h}$, almost $100 \%$ of cellular DNA remained supercoiled, which was similar with the effect of $80 \mathrm{nM}$ gimatecan (Supplement Fig. 1a). A similar phenomenon was seen when cells were treated with $30 \mathrm{nM}$ gimatecan and $10 \mu \mathrm{M}$ 

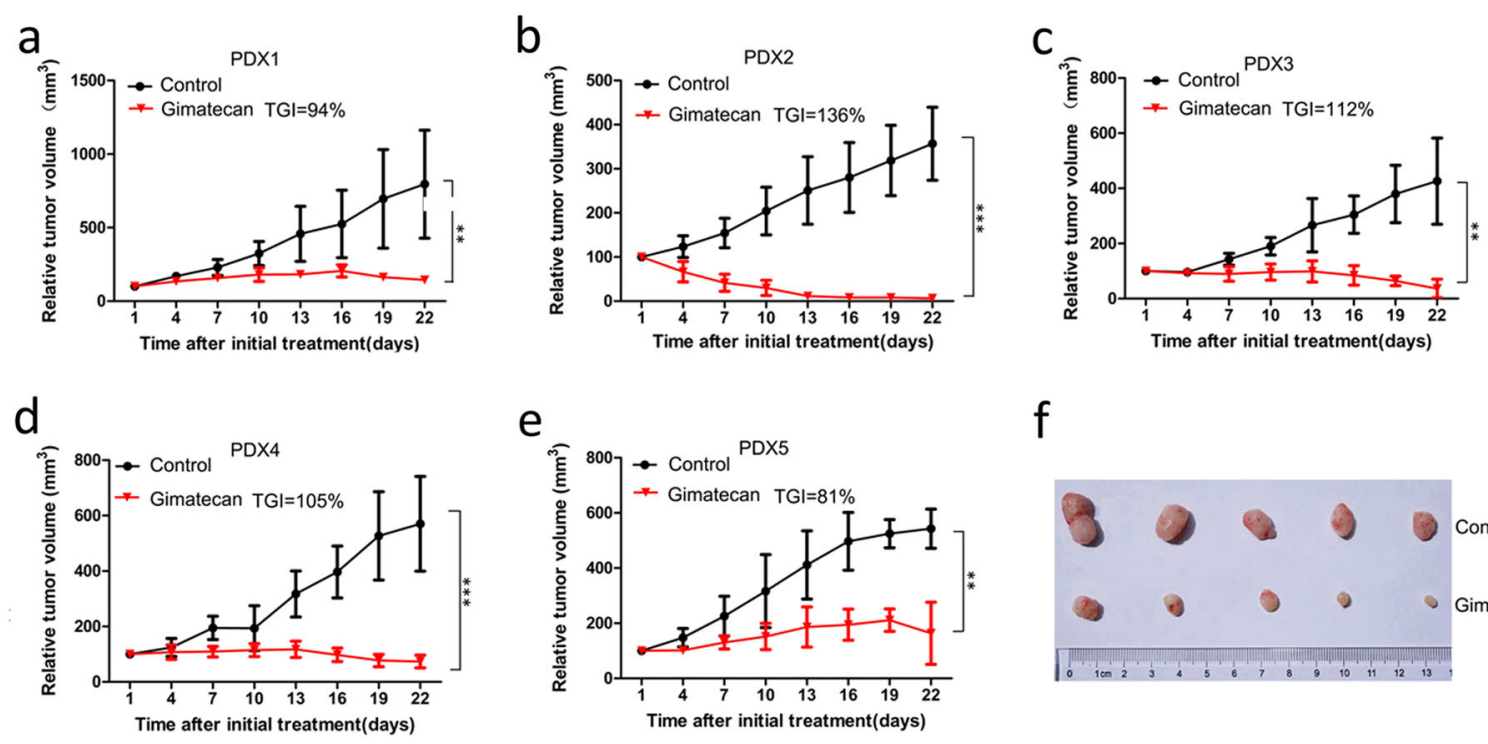

f

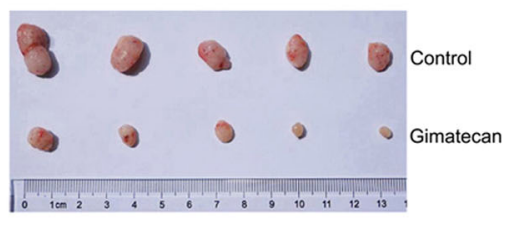

Fig. 1 Gimatecan inhibits tumor growth in patient-derived xenograft (PDX) models of esophageal squamous cell carcinoma (ESCC). a-e In vivo antitumor activity of gimatecan in ESCC PDX models. Tumors were subcutaneously engrafted and grown in NOD/SCID mice until they were $150-200 \mathrm{~mm}^{3}$ in size. Then the mice were treated with saline containing $10 \%$ DMSO or gimatecan $(0.25 \mathrm{mg} / \mathrm{kg}$, d1-d5/week, oral gavage) for 3 weeks, and tumors were measured twice a week. Tumor volume is expressed as the mean \pm SD of at least five mice in each group. Antitumor activity was analyzed using unpaired two-tailed $t$-tests, and is depicted by tumor growth inhibition. ${ }^{* *} P<0.01,{ }^{* *} P<0.001$, $\mathbf{f}$ Images of tumors dissected out from killed mice. A representative image of PDX 2 at the end of treatment is shown

irinotecan for different periods of time (Supplement Fig. 1b). Apart from the function of topoisomerase I, protein expression was also explored by Western blotting, and the results showed that gimatecan more significantly decreased the topoisomerase I expression in Eca-109 and KYSE-450 cell lines than irinotecan (Fig. 3c), as well as in the tumor tissues (Fig. 3d). And the inhibition effect of gimatecan was stronger than irinotecan both in vitro and in vivo, which was consistent with our findings of ESCC proliferation and tumorigenesis.

\section{Gimatecan induces severe DNA damage}

To characterize the DNA damage response during gimatecan treatment, the activation status and expression levels of proteins involved in DNA damage checkpoint pathways were measured in Eca-109 and KYSE-450 cell lines in vitro (Fig. 4a) and xenografts in vivo (Fig. 4b). In the event of DNA damage, those molecules are phosphorylated and activate downstream factors, such as ATM and ATR, the sensors of DNA damage; $\gamma$-H2AX and BRCA1, the mediators of DNA damage; Chk1 and Chk2, the transducers of DNA damage; and p53, the effector of DNA damage. The results showed that gimatecan treatment increased the expression of p-ATM, p-ATR, $\mathrm{p}-\mathrm{BRCA} 1, \mathrm{p}-\mathrm{H} 2 \mathrm{AX}$, and $\mathrm{p}-\mathrm{p} 53$, indicating that gimatecan could activated the DNA damage pathway at $10 \mathrm{nM}$ concentration, and the effect was stronger with the increase of gimatecan dosage to $20 \mathrm{nM}$ and $40 \mathrm{nM}$.
However, irinotecan did not induced significant effect at the same concentration. When we increased the concentration of irinotecan to $10 \mu \mathrm{M}$, similar changes were seen (Supplement Fig. 2). The results suggested that, both gimatecan and irinotecan could induced DNA damage, and the effects of gimatecan were more significant than those of irinotecan.

\section{Gimatecan induces S-phase arrest and apoptosis}

To detect how DNA damage affects cell cycle progression, we conducted cell cycle analyses in Eca-109 and KYSE-450 cell lines (Fig. 5a, b). First, we carried out propidium iodide staining to identify the cell cycle status after gimatecan and ironotecan treatment. Treatment with $10 \mathrm{nM}, 20 \mathrm{nM}$, and $30 \mathrm{nM}$ gimatecan resulted in $58.82 \% \pm 4.58 \%, 60.46 \% \pm 3.44 \%, 66.65 \% \pm 3.86 \%$ of cells in the S-phase compared with the control group of $34.75 \% \pm 2.54 \%$ in Eca-109 (all $P<0.001$ ). Similarly, treatment with $10 \mathrm{nM}, 20 \mathrm{nM}$, and $30 \mathrm{nM}$ gimatecan resulted in $40.28 \% \pm 3.87 \%, 52.88 \% \pm 4.97 \%$, and $62.13 \% \pm$ $5.34 \%$ of cells in the S-phase compared with the control group of $28.33 \% \pm 3.23 \%$ in KYSE- 450 cell lines after $4 \mathrm{~h}$ (all $P<0.001$ ). However, the same concentration of irinotecan did not induced this effect (Fig. 5a). Histograms of each phase were shown in Fig. 5b. While when we increased the dosage of irinotecan to $10 \mu \mathrm{M}$, similar $\mathrm{S}$-phase arrest induced by $30 \mathrm{nM}$ gimatecan was seen at 4 $\mathrm{h}$ and $8 \mathrm{~h}$ after treatment (Supplement Fig. 3a). 


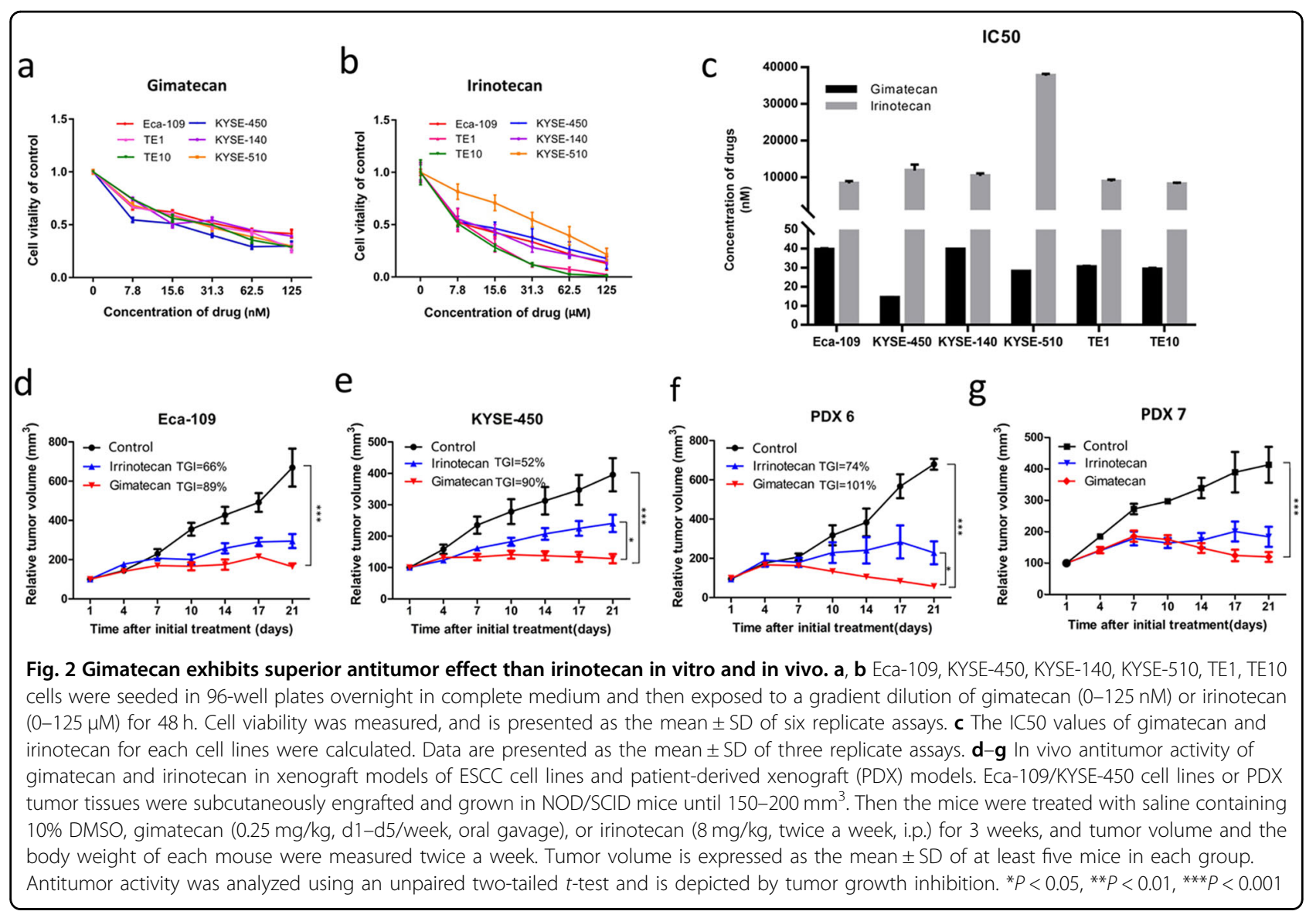

To evaluate the effects of gimatecan on cell apoptosis, we performed apoptosis analysis in Eca-109 and KYSE450 cell lines using Annexin V-PE/7-AAD staining (Fig. $5 \mathrm{c}, \mathrm{d}$ ). $10 \mathrm{nM}, 20 \mathrm{nM}$, and $30 \mathrm{nM}$ gimatecan induced a significant increase in the ratio of apoptotic cells in Eca109 cell lines $(17.76 \% \pm 0.49 \%, 21.83 \% \pm 0.66 \%, 32.75 \% \pm$ $1.02 \%$, vs $3.25 \% \pm 0.94 \%$ in the control group, all $P<0.001$ ). For KYSE- 450 cells, $10 \mathrm{nM}, 20 \mathrm{nM}$, and $30 \mathrm{nM}$ gimatecan induced $15.77 \% \pm 0.78 \%, 34.55 \% \pm 0.58 \%$, and $37.93 \% \pm 0.84 \%$ apoptotic cells respectively, compared with $5.85 \% \pm 0.1 \%$ apoptotic cells in control group (all $P<$ $0.001)$. However, irinotecan at the same concentration did not induce significant apoptosis (Fig. 5c). Column diagram of apoptotic cells were shown in Fig. 5d. While when we increased the dosage of irinotecan to $10 \mu \mathrm{M}$, significant cell apoptosis which was similar to that caused by $30 \mathrm{nM}$ gimatecan could also be seen (Supplement Fig. 3b).

To further investigate the changes in cell cycle arrest and apoptosis, the expressions of proteins related to the cell cycle, and a series of anti-apoptotic and pro-apoptotic proteins were analyzed by Western blotting. The expression of p21 was upregulated, while the expression of cyclin A and CDK2 were downregulated after treating the
Eca-109 and KYSE-450 cell lines with gimatecan. Moreover, the expression of $\mathrm{Bcl}-2$, a key regulator of the mitochondrial membrane, was downregulated, while the expression of pro-apoptotic protein Bax significantly increased after gimatecan treatment. In addition, exposure to gimatecan enhanced caspase-dependent apoptosis and activated cleaved-caspase 3 and cleaved-caspase 9. Changes induced by gimatecan were stronger than those induced by the same concentration of irinotecan (Fig. 5e). When the concentration of irinotecan was increased to $10 \mu \mathrm{M}$, the similar changes of protein expression could also be seen (Supplement Fig. 3c). Moreover, the tumor tissues of the cell line xenografts and PDX models after gimatecan and irinotecan treatment exhibited similar results of S-phase arrest and apoptosis (Fig. 5f).

Taken together, these data clearly indicate that gimatecan induce S-phase arrest, and then activate apoptosis through the pro-apoptotic signaling pathway. More importantly, gimatecan induced more obvious changes in cell cycle arrest and apoptosis, which was also in accordance with the suppression of tumor growth in vivo.

In summary, our data demonstrate that the anticancer activity of gimatecan is mediated through topoisomerase inhibition and subsequent induction of DNA damage. 


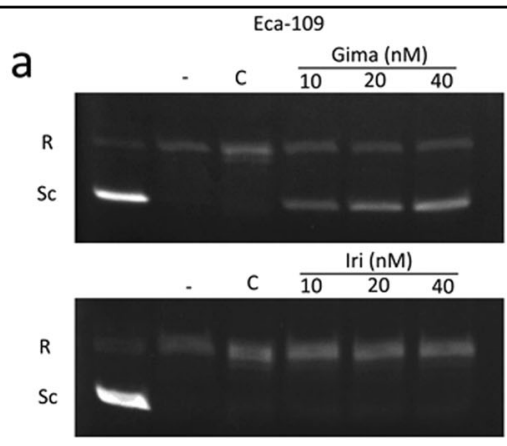

C

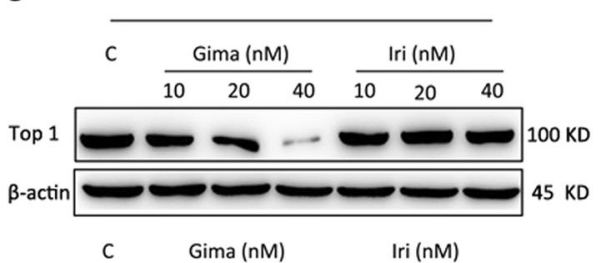
C $\frac{\text { Gima }(\mathrm{nM})}{10 \quad 20 \quad 40} \quad \frac{\operatorname{lr}(\mathrm{nM})}{10 \quad 20 \quad 40}$

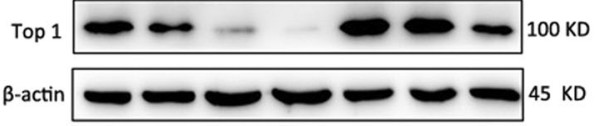

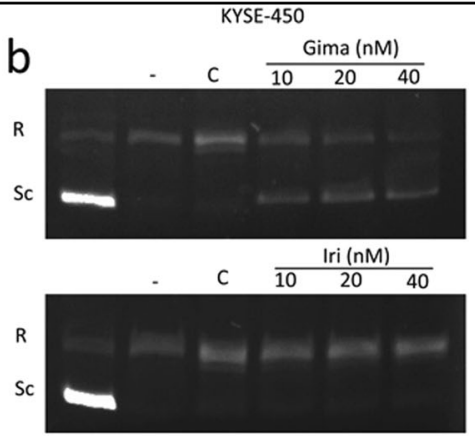

d tumor tissue

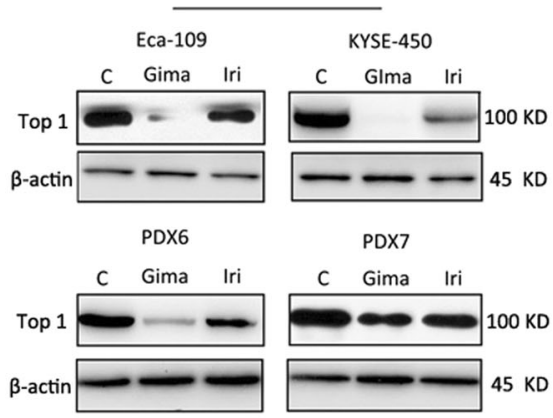

Fig. 3 Gimatecan reduces topoisomerase I specific activity and suppress topoisomerase I expression in ESCC. $\mathbf{a}$, $\mathbf{b}$ Experiment of topoisomerase I activity using different assay: Eca-109 and KYSE-450 cell lines were exposed to serial dilutions (10 nM, 20 nM, and 40 nM) of gimatecan and irinotecan for $2 \mathrm{~h}$, and $20 \mu \mathrm{L}$ of reaction containing nucleoli extract protein was incubated with supercoiled DNA for 30 min. Lane 1, supercoiled DNA only. Lane 2, relaxed DNA used as negative control. Lanes 3-5, supercoiled DNA, and nucleoli extract protein of cells treated with different concentration of gimatecan or irinotecan for $2 \mathrm{~h} ; \mathbf{c}$, $\mathbf{d}$ the expression of topoisomerase 1 was assessed by Western blotting in vitro and in vivo. Eca-109 and KYSE-450 cell lines were exposed to $10 \mathrm{nM}, 20 \mathrm{nM}$, and $40 \mathrm{nM}$ gimatecan or irinotecan for $48 \mathrm{~h}$, and harvested at 70-80\% confluence. For in vivo experiment, the mice were killed and tumor tissues of Eca-109 and KYSE-450 cell line xenografts and PDX models were harvested at the end of treatment. Total protein was extracted from harvested cell lines or tumor tissues, and the expression of topoisomerase 1 was assessed by Western blotting

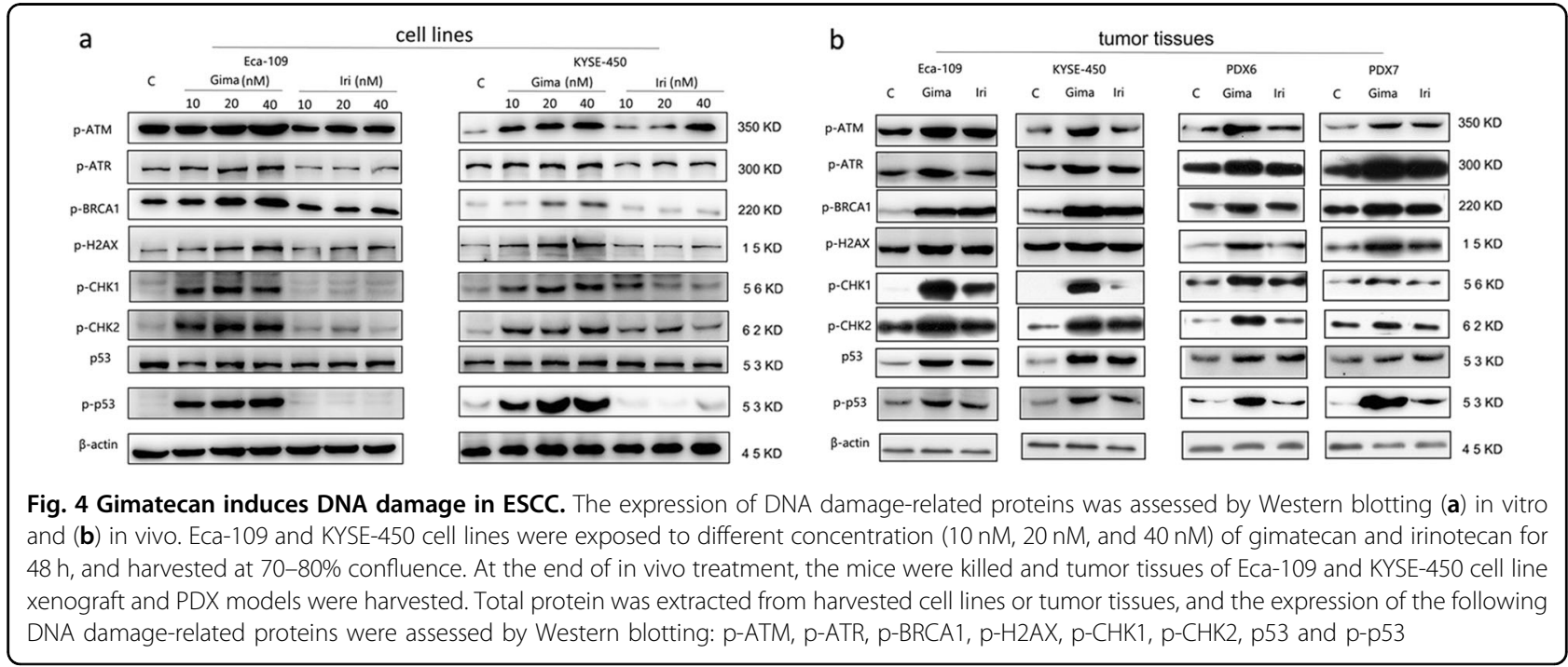




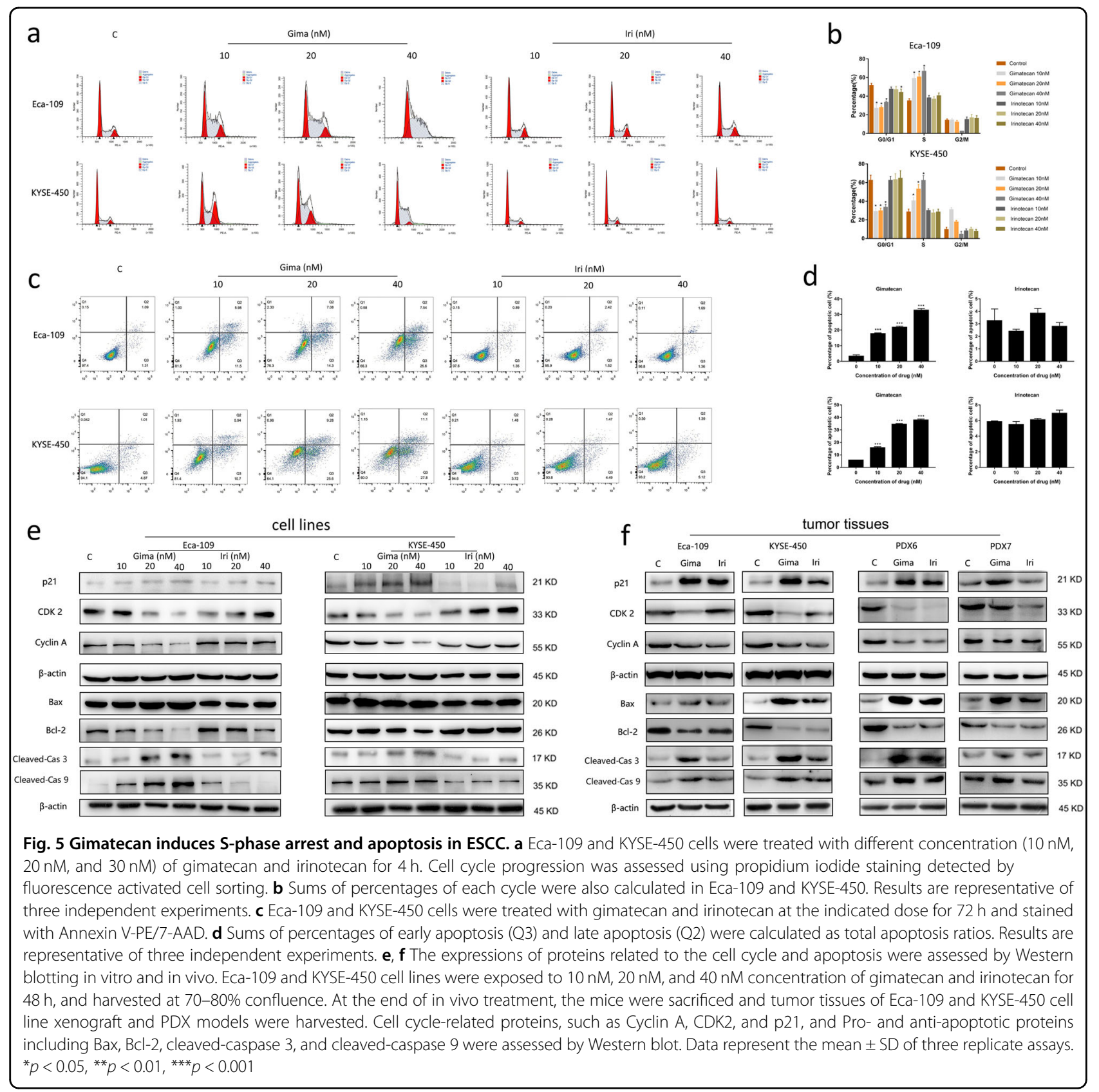

Then DNA damage response signaling activates p53 and leads to the accumulation of p21 and S-phase cell cycle arrest and induction of apoptosis in ESCC, as depicted and summarized in Fig. 6. The mechanism of gimatecan is similar to irinotecan, but gimatecan induces stronger effects.

\section{Discussion}

We evaluated the chemotherapeutic effects and mechanisms of action of gimatecan, a lipophilic oral camptothecin analog, by comparing it to a first-line clinical agent, irinotecan. Gimatecan inhibited the proliferation of multiple EC cell lines, suppresses topoisomerase I activity, induced DNA damage, arrest cell cycle, caused cell apoptosis, and repressed tumor growth in mice models more significantly than irinotecan, demonstrating that it might potentially be a more powerful anticancer agent than irinotecan.

Exposure of cells to topoisomerase inhibitors could stabilize the cleavable complex. The appearance of collision between stable topoisomerase I-drug-DNA complexes and replication forks or transcription complexes generally induces lethal double-strand breaks in $\mathrm{DNA}^{24}$. The DNA breaks lead to DNA damage, which is the 


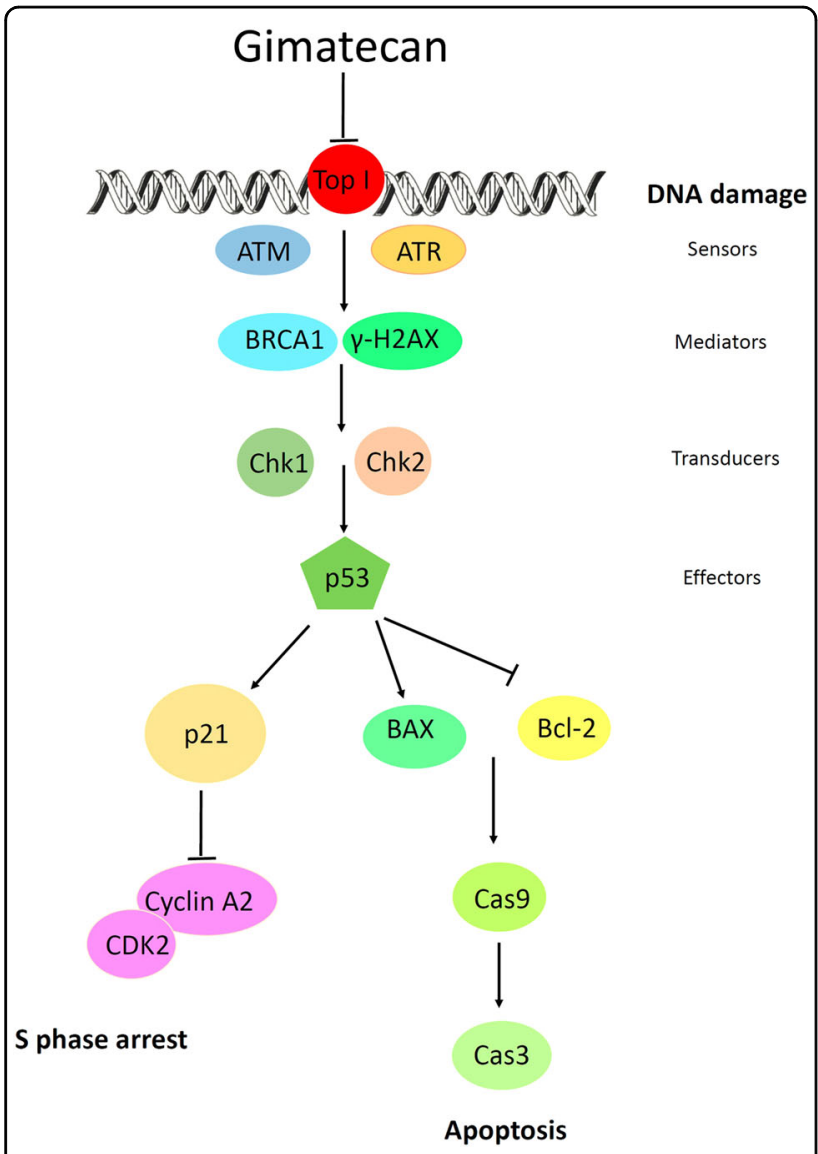

Fig. 6 Proposed mechanisms of gimatecan-induced DNA damage, Sphase arrest, and apoptosis in ESCC

central mechanism of antitumor activity ${ }^{25,26}$. DNA damage triggers activation of DNA damage-response elements, such as ATM and ATR. We found that treatment with gimatecan induces phosphorylation of both ATM and ATR proteins in ESCC both in vitro and in vivo. Activated ATM and ATR, either directly or through sequential steps, phosphorylate the downstream proteins BRCA1, H2AX, Chk1, and Chk2, and subsequently affect downstream factors involved in cell cycle progression and cell survival ${ }^{27-29}$. The tumor suppressor protein p53 is a crucial component of cellular machinery that regulates various signaling pathways including DNA damage, the cell cycle, and apoptosis ${ }^{30-32}$. Under stressed conditions, such as induction of DNA damage, post-translational modifications such as phosphorylation and acetylation may also play a role in enhanced p53 levels ${ }^{30,32}$. In our study, increased expression of phosphorylated p53 was found after gimatecan treatment, which is consistent with this viewpoint.

The increased expression of p53 after CPT treatment results in cell cycle arrest ${ }^{33-35}$. Irinotecan may induce cell cycle arrest in different phases including the S-phase and
G2/M-phase in testicular cancer ${ }^{36}$, colon cancer $^{37}$, and non-small cell lung cancer ${ }^{38}$. Gimatecan induces S-phase arrest in bladder carcinoma, ovarian carcinoma, and melanoma $^{21,39}$. In our study, both gimatecan and irinotecan induced intra-S-phase arrest in EC in vitro and in vivo. These findings are similar to previous studies, except for a small discrepancy that might be due to the inherent heterogeneity of different tumor types, because the multiple signals that regulate the cell cycle can be cell type-specific. We hypothesized that activation of p53 functioned as a transcriptional activator of some target genes including p21 WAF1. Activated p21 proteins interact with $\mathrm{CDK} 2 /$ cyclin $\mathrm{A} 2$ and inhibit binding of CDK2/cyclin A, resulting in S-phase arrest.

Activated p53 may induce not only cell cycle arrest but also apoptosis activation or cellular senescence ${ }^{30,31}$. Elevation of p53 induces Bax expression, downregulates the anti-apoptotic protein $\mathrm{Bcl}-2$, and activates the caspase 3/7/9-dependent pathway, which is associated with the inhibition of tumor cell growth ${ }^{31}$. In our study, the expression of Bcl-2 protein significantly decreased, while cleaved-caspase 3 and cleaved-caspase 9 increased after gimatecan treatment, which indicates that gimatecan induced apoptosis. Cellular senescence was also evaluated in vitro after gimatecan treatment for $24 \mathrm{~h}, 48 \mathrm{~h}, 72 \mathrm{~h}$, and $96 \mathrm{~h}$ using the senescence-associated beta galactosidase detection solution, and no senescence was observed.

Gimatecan retained stronger potency in antitumor efficacy compared to irinotecan in ESCC in this study. Similar results have also been reported in prostate carcinoma ${ }^{40}$ and neuroblastoma ${ }^{41}$, and the modified structure contributes to it a lot. Among a series of seven substituted camptothecins, gimatecan was developed with lipophilic modification at position C-7. The modification enhances stability of the drug-enzyme-DNA complex and lactone ring, and ensures rapid agent intake and stable drug interactions with intracellular targets. Furthermore, gimatecan has a favorable tissue distribution, with particular reference to the ability to cross the blood-brain barrier as a consequence of lipophilic modification, which is also supported by in vivo distribution studies in glioma preclinical models ${ }^{42}$. It is likely that the cytotoxic potency exhibited by gimatecan is the result of the combination of these features. In additional, oral administration is much more convenient than intravenous injection, which is the method used to administer irinotecan. Intestinal absorption is crucial for oral treatment, and could ensure a high drug concentration in the liver, which is a common site of metastasis for ESCC. In contrast to irinotecan and topotecan, gimatecan is not a substrate for breast cancer resistance protein (BCRP), an efflux pump for the multidrug resistance P-glycoprotein ${ }^{22}$, so gimatecan is able to overcome cellular resistance in mitoxantrone-selected cell lines characterized by high levels of BCRP expression ${ }^{43}$. 
In summary, our study demonstrates that by suppressing topoisomerase I, inducing S-phase cell cycle arrest and DNA damage, and promoting apoptosis, the new camptothecin analog gimatecan strongly inhibits ESCC growth both in vitro and in vivo. In addition, it shows improved antitumor efficacy compared to the current first-line agent, irinotecan, which indicates that this new oral medicine may serve as a better clinical option for ESCC-targeted therapy.

\section{Materials and methods Agents}

Gimatecan (7-t-butoxyiminomethylcamptothecin, purity $\geq 99.9 \%$ ) was synthesized and kindly provided by Zhaoke Pharmaceutical Ltd (Hefei, China) ${ }^{22,42}$. The chemical agent was dissolved in dimethylsulfoxide (DMSO) and stored at $-80{ }^{\circ} \mathrm{C}$. Irinotecan (purity $\geq 99.9 \%$ ) was purchased from Jiangsu Hengrui Medicine Co., Ltd (Jiangsu, China), and was dissolved in sterile distilled water before use.

\section{Cell lines and cell viability assay}

Esophageal squamous carcinoma cell lines, including EC-109, KYSE450, KYSE-140, KYSE-510, and TE-1, TE10 , were used in this study. Cells were maintained in RPMI 1640 media (Gibco-BRL, MD, USA) supplemented with $10 \%$ fetal bovine serum (FBS, Gibco) and $1 \%$ penicillin-streptomycin (Gibco) in a humidified incubator $\left(37^{\circ} \mathrm{C}\right)$ with $5 \% \mathrm{CO}_{2}$.

Cells were seeded into 96-well plates at a density of $2-5 \times 10^{3}$ cells/well overnight in complete medium, and then were treated the next day with gimatecan $(0-1000$ $\mathrm{nM})$ or irinotecan $\left(0-1 \times 10^{6} \mathrm{nM}\right)$. After $48 \mathrm{~h}$ incubation, cell viability was measured using a Cell Counting Kit-8 (CCK8) assay (Dojindo, Kumamoto, Japan) according to the manufacturer's instructions. Absorbance was measured at $450 \mathrm{~nm}$ using a spectrophotometer. All experiments were repeated and read at least three times. IC50 was defined as the concentration of drug causing a 50\% reduction in cell number compared to that of untreated controls. The concentration of $30 \mathrm{nM}$ gimatecan or $10 \times$ $10^{3} \mathrm{nM}$ irinotecan was applied for in vitro experiments according to the IC50 values.

\section{DNA topoisomerase I activity assay}

DNA strand breakage induced by topoisomerase I was evaluated by the conversion of the double-stranded supercoiled DNA to a relaxed form. Cells were treated with gimatecan or irinotecan in a dosage-dependent manner for $2 \mathrm{~h}$, and a time-dependent manner at $30 \mathrm{nM}$ gimatecan and $10 \times 10^{3}$ irinotecan, and then nucleoli were isolated. Topoisomerase I activity was assessed in isolated nucleoli using an experimental kit (TopoGen Inc., Port Orange, FL) according to the manufacturer's instructions.
Briefly, a $20 \mu \mathrm{L}$ reaction containing nucleoli extract protein was incubated with supercoiled DNA (100 ng, provided by the kit) in $1 \times$ reaction buffer $(10 \mathrm{mM}$ Tris- $\mathrm{HCl}$, $\mathrm{pH}$ 7.9, $1 \mathrm{mM}$ EDTA, $1.5 \mathrm{M} \mathrm{NaCl}, 0.1 \mathrm{mM}$ spermidine, $50 \%$ glycerol, $0.1 \% \mathrm{BSA}$ ) for $30 \mathrm{~min}$ at $37^{\circ} \mathrm{C}$ and terminated using stop loading buffer ( $1 \%$ sarkosyl, $0.025 \%$ bromophenol blue, $5 \%$ glycerol). Then the samples were loaded on a $1 \%$ agarose gel and run in $1 \times$ TAE $(40 \mathrm{mM}$ Tris-Acetate, $1 \mathrm{mM}$ EDTA, $\mathrm{pH}$ 8.3) buffer for $80 \mathrm{~V}$ for 30 min. The gels were stained with $0.5 \mathrm{mg} / \mathrm{mL}$ ethidium bromide for $30 \mathrm{~min}$ and then destained in distilled water for $20 \mathrm{~min}$. After that, the gels were photographed using a UV transilluminator (Bio-Rad, Hercules, CA).

\section{Flow cytometry}

To analyze the changes in cell cycle progression, cell pellets were harvested after exposure to gimatecan or irinotecan for $0 \mathrm{~h}, 4 \mathrm{~h}, 8 \mathrm{~h}$, and then fixed in $70 \%$ cold ethanol at $4{ }^{\circ} \mathrm{C}$ overnight. Then the fixed cells were stained with $50 \mu \mathrm{g} / \mathrm{mL}$ propidium iodide (BD Biosciences), and incubated at room temperature in darkness for $30 \mathrm{~min}$. Flow cytometry was performed using a FACS Calibur system (BD Biosciences) and data were analyzed by ModFit 4.0 software (BD Biosciences).

For cell apoptosis analysis, cells were exposed to gimatecan or irinotecan for $24 \mathrm{~h}, 48 \mathrm{~h}$, or $72 \mathrm{~h}$, and then stained with Annexin V-phycoerythrin PE and 7-aminoactinomycin (7-AAD) (BD Biosciences, Erembodegem, Belgium) at room temperature in the dark for $15 \mathrm{~min}$. The flow cytometric analyses were conducted within $30 \mathrm{~min}$. Cell apoptotic data were analyzed using the FlowJo software (TreeStar, Inc., Ashland, OR).

\section{Antitumor activity studies}

In vivo antitumor experiments were conducted using 6- to 8-week-old female NOD/SCID mice (Beijing Vital River Laboratory Animal Technology Co., Ltd.). The mice were maintained in laminar flow rooms, with constant temperature and humidity and free access to food and water. All animal experiments were performed according to the animal experimental guidelines of Peking University Cancer Hospital and followed internationally recognized Animal Research: Reporting of In Vivo Experiments guidelines. Tumor cell line xenografts were established by subcutaneous injection of $2 \times 10^{6}$ cells from in vitro cultures. Patient-derived xenograft (PDX) models were established in our lab according to previous studies $^{44}$. Tumors were measured with fine calipers twice a week. When tumors were $\sim 150-200 \mathrm{~mm}^{3}$, mice were randomly assigned into the treatment group or the control group (5-6 per group). The mice in the control group were treated with saline containing $10 \%$ DMSO. The mice in the treatment group were given gimatecan $(0.25 \mathrm{mg} / \mathrm{kg}$, $\mathrm{d} 1-\mathrm{d} 5 /$ week, oral gavage $)$ or irinotecan $(40 \mathrm{mg} / \mathrm{kg}$ twice 
weekly, intraperitoneal injection) according to previous reports $^{45,46}$. All of the animals were treated for 3 weeks. At the end of treatment, the tumor tissues of PDX models were harvested and kept at $-80^{\circ} \mathrm{C}$.

Parameters related to tumor growth were used as previously reported ${ }^{44}$ : Tumor volume $=(\text { Length } \times \text { Width })^{2} / 2$; Tumor growth inhibition $(\mathrm{TGI})=\Delta T / \Delta C \times 100 \% ;(\Delta T=$ tumor volume change in the treatment group on the final day of the study, $\Delta C=$ tumor volume change in the control group on the final day of the study).

\section{Western blots}

Eca-109 and KYSE-450 cells were seeded in 6-well plates with complete medium. Twenty-fourth after planting, cells were treated with $30 \mathrm{nM}$ gimatecan or $10 \times$ $10^{3} \mathrm{nM}$ irinotecan for $48 \mathrm{~h}$ and then harvested. The cell pellets and tumor tissues of xenografts were lysed and protein concentrations were measured. Protein samples were diluted to equal concentrations $(10 \mu \mathrm{g} / \mu \mathrm{L})$, and separated by $8-12 \%$ SDS-PAGE. After being transferred onto nitro-cellulose membranes (GE Healthcare, Piscataway, NJ), samples were incubated with corresponding primary antibodies in $5 \% \mathrm{BSA}$ at $4{ }^{\circ} \mathrm{C}$ overnight, then washed and incubated in secondary antibodies at room temperature for $1 \mathrm{~h}$. Antibodies in this study included topoisomerase I, Cyclin A2, (Santa Cruz, CA, USA), p-ATM (Ser1981), p-ATR (Ser428), p-BRCA1 (Ser1524), p-Chk1(Ser345), p-Chk2 (Thr68), p-H2AX (Ser139), p-p53 (Ser15), p-CDC25C (Ser216), p21(Waf1/Cip1), p53, CDK2, Bax, Bcl-2, Cleaved-Caspase 3, Cleaved-Caspase 9 (Cell Signaling Technology, Boston, MA, USA), and $\beta$-actin (Sigma-Aldrich, USA). Proteins were visualized using ECLplus Western Blotting Detection Reagents (GE Healthcare).

\section{Statistical analysis}

Statistical analyses, including one-way analysis of variance and the two-tailed Student's $t$-test, were performed using SPSS 21.0 software (Inc., Chicago, IL, USA). Data are expressed as the mean \pm standard deviation in each case. $P<0.05$ was considered statistically significant.

\section{Acknowledgements}

This work was supported by Beijing Municipal Administration of Hospital Program (QML20151002), Beijing Municipal Senior Technical Training Plan in Health System (2015-3-073). The authors thank Zhaoke Pharmaceutical Ltd (Hefei, China) for providing the drug of gimatecan. The authors thank Xiangqian Kong in Johns Hopkins Medicine \{Pratesi, 2002 \# 321\} for providing valuable advices.

\section{Conflict of interest}

The authors declare that they have no conflict of interest.

\section{Publisher's note}

Springer Nature remains neutral with regard to jurisdictional claims in published maps and institutional affiliations.
Supplementary Information accompanies this paper at https://doi.org/ 10.1038/s41419-018-0700-0.

Received: 10 February 2018 Revised: 11 May 2018 Accepted: 14 May 2018 Published online: 31 May 2018

\section{References}

1. Chen, W. et al. Cancer statistics in China, 2015. CA Cancer J. Clin. 66, 115-132 (2016).

2. Malhotra, G. K. et al. Global trends in esophageal cancer. J. Surg. Oncol. 115 564-579 (2017)

3. Zeng, $\mathrm{H}$. et al. Cancer survival in China, 2003-2005: a population-based study. Int. J. Cancer 136, 1921-1930 (2015).

4. Dubecz, A. et al. Temporal trends in long-term survival and cure rates in esophageal cancer: a SEER database analysis. J. Thorac. Oncol. 7, 443-447 (2012).

5. Hsiang, Y. H., Hertzberg, R., Hecht, S. \& Liu, L. F. Camptothecin induces proteinlinked DNA breaks via mammalian DNA topoisomerase I. J. Biol. Chem. 260 14873-14878 (1985)

6. Hsiang, Y. H., Lihou, M. G. \& Liu, L. F. Arrest of replication forks by drugstabilized topoisomerase I-DNA cleavable complexes as a mechanism of cell killing by camptothecin. Cancer Res. 49, 5077-5082 (1989).

7. Takaoka, T. et al. Panitumumab in combination with irinotecan plus S-1 (IRIS) as second-line therapy for metastatic colorectal cancer. Cancer Chemother. Pharmacol. 78, 397-403 (2016).

8. Volovat, S. R., Volovat, C., Negru, S. M., Danciu, M. \& Scripcariu, V. The efficacy and safety of hepatic arterial infusion of oxaliplatin plus intravenous irinotecan, leucovorin and fluorouracil in colorectal cancer with inoperable hepatic metastasis. J. Chemother. 28, 235-241 (2016).

9. William, W. N. Jr. et al. Phase I trial of weekly topotecan and gemcitabine in patients with solid tumors. Am. J. Clin. Oncol. 32, 15-19 (2009).

10. White, S. C. et al. Phase II study of oral topotecan in advanced non-small cell lung cancer. Clin. Cancer Res. 6, 868-873 (2000).

11. West, W. et al. Phase I study of paclitaxel and topotecan for the first-line treatment of extensive-stage small cell lung cancer. Oncologist 8, 76-82 (2003).

12. Goto, K. et al. Combined chemotherapy with cisplatin, etoposide, and irinotecan versus topotecan alone as second-line treatment for patients with sensitive relapsed small-cell lung cancer (JCOG0605): a multicentre, open-label, randomised phase 3 trial. Lancet Oncol. 17, 1147-1157 (2016).

13. Thallinger, C. M., Raderer, M. \& Hejna, M. Esophageal cancer: a critical evaluation of systemic second-line therapy. J. Clin. Oncol. 29, 4709-4714 (2011).

14. Busino, L. et al. Degradation of Cdc25A by beta-TrCP during $S$ phase and in response to DNA damage. Nature 426, 87-91 (2003).

15. Ilson, D. H. et al. Phase II trial of weekly irinotecan plus cisplatin in advanced esophageal cancer. J. Clin. Oncol. 17, 3270-3275 (1999).

16. Gerrits, C. J., de Jonge, M. J., Schellens, J. H., Stoter, G. \& Verweij, J. Topoisomerase I inhibitors: the relevance of prolonged exposure for present clinical development. Br. J. Cancer 76, 952-962 (1997).

17. Houghton, P. J. et al. Evaluation of 9-dimethylaminomethyl-10hydroxycamptothecin against xenografts derived from adult and childhood solid tumors. Cancer Chemother. Pharmacol. 31, 229-239 (1992).

18. Polizzi, D. et al. A novel taxane with improved tolerability and therapeutic activity in a panel of human tumor xenografts. Cancer Res. 59, 1036-1040 (1999).

19. Dallavalle, S. et al. Novel 7-substituted camptothecins with potent antitumor activity. J. Med. Chem. 43, 3963-3969 (2000).

20. Zunino, F. et al. Current status and perspectives in the development of camptothecins. Curr. Pharm. Des. 8, 2505-2520 (2002).

21. Pratesi, G., Beretta, G. L. \& Zunino, F. Gimatecan, a novel camptothecin with a promising preclinical profile. Anti-Cancer Drugs 15, 545-552 (2004).

22. De Cesare, M. et al. Potent antitumor activity and improved pharmacological profile of ST1481, a novel 7-substituted camptothecin. Cancer Res. 61, 7189-7195 (2001)

23. Pratesi, G. et al. Pattern of antitumor activity of a novel camptothecin, ST1481, in a large panel of human tumor xenografts. Clin. Cancer Res. 8, 3904-3909 (2002).

24. Beretta, G. L., Perego, P. \& Zunino, F. Targeting topoisomerase I: molecular mechanisms and cellular determinants of response to topoisomerase I inhibitors. Expert Opin. Ther. Targets 12, 1243-1256 (2008). 
25. Pommier, Y., Leo, E., Zhang, H. \& Marchand, C. DNA topoisomerases and their poisoning by anticancer and antibacterial drugs. Chem. Biol. 17, 421-433 (2010).

26. Wang, J. C. Cellular roles of DNA topoisomerases: a molecular perspective. Nat. Rev. Mol. Cell Biol. 3, 430-440 (2002).

27. Marechal, A. \& Zou, L. DNA damage sensing by the ATM and ATR kinases. Cold Spring Harb. Perspect. Biol. 5, a012716 (2013). https://www. ncbi.nlm.nih.gov/pmc/articles/PMC3753707/

28. Gottifredi, V. \& Prives, C. The S phase checkpoint: when the crowd meets at the fork. Semin. Cell Dev. Biol. 16, 355-368 (2005)

29. Sancar, A., Lindsey-Boltz, L. A., Unsal-Kacmaz, K. \& Linn, S. Molecular mechanisms of mammalian DNA repair and the DNA damage checkpoints. Annu. Rev. Biochem. 73, 39-85 (2004).

30. Speidel, D. The role of DNA damage responses in p53 biology. Arch. Toxicol. 89, 501-517 (2015).

31. Chen, J. The cell-cycle arrest and apoptotic functions of p53 in tumor initiation and progression. Cold Spring Harb. Perspect. Med. 6, a026104 (2016).

32. Mazouzi, A., Velimezi, G. \& Loizou, J. I. DNA replication stress: causes, resolution and disease. Exp. Cell Res. 329, 85-93 (2014).

33. Cliby, W. A., Lewis, K. A., Lilly, K. K. \& Kaufmann, S. H. S phase and G2 arrests induced by topoisomerase I poisons are dependent on ATR kinase function. J. Biol. Chem. 277, 1599-1606 (2002).

34. Lin, R. W. et al. CFS-1686 causes cell cycle arrest at intra-S phase by interference of interaction of topoisomerase 1 with DNA. PLOS ONE 9, e113832 (2014).

35. Wu, N. et al. A novel DNA topoisomerase I inhibitor with different mechanism from camptothecin induces G2/M phase cell cycle arrest to K562 cells. Biochemistry 49, 10131-10136 (2010).

36. Ueno, M., Nonaka, S., Yamazaki, R., Deguchi, N. \& Murai, M. SN-38 induces cell cycle arrest and apoptosis in human testicular cancer. Eur. Urol. 42, 390-397 (2002).
37. Abu-Sanad, A. et al. Simultaneous inhibition of ATR and PARP sensitizes colon cancer cell lines to irinotecan. Front. Pharmacol. 6, 147 (2015).

38. Maurya, D. K., Ayuzawa, R., Doi, C., Troyer, D. \& Tamura, M. Topoisomerase inhibitor SN-38 effectively attenuates growth of human non-small cell lung cancer cell lines in vitro and in vivo. J. Environ. Pathol. Toxicol. Oncol. 30, 1-10 (2011).

39. Ulivi, P. et al. Cellular basis of antiproliferative and antitumor activity of the novel camptothecin derivative, gimatecan, in bladder carcinoma models. Neoplasia 7, 152-161 (2005)

40. Di Francesco, A. M. et al. The novel lipophilic camptothecin analogue gimatecan is very active in vitro in human neuroblastoma: a comparative study with SN38 and topotecan. Biochem. Pharmacol. 70, 1125-1136 (2005).

41. Bom, D. et al. The novel silatecan 7-tert-butyldimethylsilyl-10-hydroxycamptothecin displays high lipophilicity, improved human blood stability, and potent anticancer activity. J. Med. Chem. 43, 3970-3980 (2000).

42. Pollack, I. F. et al. Potent topoisomerase I inhibition by novel silatecans eliminates glioma proliferation in vitro and in vivo. Cancer Res. 59, 4898-4905 (1999).

43. Perego, P. et al. A novel 7-modified camptothecin analog overcomes breast cancer resistance protein-associated resistance in a mitoxantrone-selected colon carcinoma cell line. Cancer Res. 61, 6034-6037 (2001).

44. Zou, J. et al. Establishment and genomic characterizations of patient-derived esophageal squamous cell carcinoma xenograft models using biopsies for treatment optimization. J. Transl. Med. 16, 15 (2018).

45. Ottolino-Perry, K. et al. Oncolytic vaccinia virus synergizes with irinotecan in colorectal cancer. Mol. Oncol. 9, 1539-1552 (2015).

46. Mazard, T. et al. Sorafenib overcomes irinotecan resistance in colorectal cancer by inhibiting the ABCG2 drug-efflux pump. Mol. Cancer Ther. 12, 2121-2134 (2013). 